Musées, Patrimoine et Culture scientifiques et techniques

$132 \mid 2010$

novembre - décembre 2010

\title{
La Société du Vieux-Port de Montréal (SVPM) et sa fréquentation
}

\section{Haja Ramahatra}

\section{OpenEdition \\ Journals}

Édition électronique

URL : http://journals.openedition.org/ocim/405

DOI : 10.4000/ocim.405

ISSN : 2108-646X

\section{Éditeur}

OCIM

Édition imprimée

Date de publication : 1 novembre 2010

Pagination : $56-60$

ISSN : 0994-1908

\section{Référence électronique}

Haja Ramahatra, «La Société du Vieux-Port de Montréal (SVPM) et sa fréquentation », La Lettre de I'OCIM [En ligne], 132 | 2010, mis en ligne le 01 novembre 2012, consulté le 19 avril 2019. URL : http:// journals.openedition.org/ocim/405; DOI : 10.4000/ocim.405

Ce document a été généré automatiquement le 19 avril 2019.

Tous droits réservés 


\title{
La Société du Vieux-Port de Montréal (SVPM) et sa fréquentation
}

\author{
Haja Ramahatra
}

1 La Société du Vieux-Port de Montréal inc. a été créée en 1981 par le gouvernement du Canada avec le mandat « de développer et de promouvoir le territoire du Vieux-Port de Montréal ainsi que d'administrer et de gérer les biens de Sa Majesté ». Vaste site récréotouristique et culturel urbain d'une superficie de 44 hectares, ce territoire s'étend sur 2,3 kilomètres le long du fleuve Saint-Laurent, en bordure du Vieux-Montréal. Le site comprend deux unités d'affaires : les Quais du Vieux-Port de Montréal (site extérieur) et le Centre des Sciences de Montréal (CSM) ${ }^{1}$.

2 Montréal accueille annuellement 15 millions de visiteurs en moyenne, dont 7 millions de touristes ${ }^{2}$. Ces visiteurs apprécient plus particulièrement la visite des sites et établissements à caractère historique, muséal ou patrimonial, et la visite du quartier historique constitue pour la grande majorité d'entre eux un « incontournable » ${ }^{3}$.

Ce bassin naturel de visiteurs est entretenu à longueur d'année par l'attrait du quartier historique ainsi que par les activités de programmation (Cirque du Soleil, spectacles divers, feux d'artifice, visites de bateaux historiques, patinoire...), récréotouristiques (croisières, balade historique...) et commerciales (restaurants, terrasses, boutiques) offertes sur le site qui contribuent à maintenir les Quais du Vieux-Port au troisième rang des sites récréotouristiques au sein de la grande région de Montréal avec un achalandage de 5,2 millions de visiteurs et un taux de notoriété de $96 \%$ pour l'année $2009{ }^{4}$.

Le Centre des Sciences de Montréal (CSM), situé sur le site récréotouristique, bénéficie également de l'achalandage des Quais du Vieux-Port et indirectement de celui du quartier historique, bien que sa clientèle soit plus ciblée et plus locale. Depuis son ouverture en 2000, le CSM a accueilli plus de 7 millions de visiteurs, avec un achalandage de 817000 en 2009 et un taux de notoriété de $70 \%$ qui le place en tête dans la catégorie des centres de divertissement à caractère scientifique de la grande région de Montréal, devant le Planétarium et le Biodôme ${ }^{5}$. 
5 L'exercice d'établir une estimation de l'achalandage du site, soit la fréquentation, des Quais du Vieux-Port s'avère être un véritable défi compte tenu de l'étendue du territoire, du nombre d'accès possible et de la nature même du site (lieu extérieur ouvert et accessible au grand public). Connaître l'achalandage du site extérieur demeure un enjeu pour les Quais du Vieux-Port et pour la SVPM en général car de cette information découle les constats et les analyses qui influencent autant les opérations que le déroulement et la planification des activités de programmation et du marketing ainsi que le suivi des indicateurs de performance dans le cadre des exercices prévisionnels.

6 C'est dans ce contexte que la SVPM a décidé, vers la fin des années 1990, de faire appel à une firme chargée de définir une méthodologie permettant l'estimation de l'achalandage sur le site extérieur, et ce, en tenant compte des contraintes particulières du site. Cette méthodologie s'appuie principalement sur une modélisation mathématique de la fréquentation du site qui est alimentée autant par une observation in situ que par des données internes et externes. Il s'agit d'une approche méthodologique hybride qui s'adapte au fil du temps et qui, jusqu'à présent, a toujours répondu aux besoins de la SVPM et de ses groupes d'intérêts.

\section{La disparité des méthodes d'estimation de mesure de la fréquentation}

7 De manière générale, afin qu'elle soit statistiquement fiable, une estimation de l'achalandage nécessite le recours à des méthodes systématiques de dénombrement et de pointage du flux de visiteurs ou de participants. Cependant, si les résultats des différentes méthodes permettent d'obtenir des estimations relativement fiables (dans la mesure où les exigences méthodologiques ont été respectées), le niveau d'implication sur le plan des ressources humaines et financières constitue le facteur déterminant en ce qui concerne le choix final de la méthode qui sera mise en œuvre par l'organisme ou le promoteur d'événement. De plus, la nature particulière de chaque organisme ou événement nécessite une approche méthodologique au cas par cas, ce qui rend impossible l'instauration d'une méthode d'estimation unique ou standardisée qui puisse convenir à l'ensemble des intervenants.

8 Au Québec, dans le secteur touristique et culturel, le choix des méthodes d'estimation et d'évaluation de l'achalandage ainsi que la divulgation (ou diffusion) des résultats restent à la discrétion des établissements ou des promoteurs d'événements. Toutefois, pour l'intervenant ou l'individu qui bénéficie d'une subvention du gouvernement, l'octroi ou le renouvellement d'une aide financière est obligatoirement assorti de la mise en place d'un certain nombre d'indicateurs de performance, entre autres la mesure de l'achalandage ou de la fréquentation. De ce fait, le ministère du Tourisme du Québec, après avoir consulté des firmes spécialisées en sondage et des intervenants touristiques, a produit un document faisant une synthèse des méthodes reconnues et largement utilisées pour effectuer une estimation de l'achalandage.

9 Comme le démontre le tableau ci-joint, le choix d'une méthode d'estimation de l'achalandage repose principalement sur trois facteurs :

- le type d'établissement ou la nature de l'événement: entrée gratuite ou payante, pour le grand public ou pour une clientèle spécifique, d'une durée limitée ou sur toute l'année... 
- l'envergure et l'accès du site (lieu physique) : occupation spatiale, nombre d'entrées ou voies d'accès, espace ouvert ou fermé...

- la capacité de l'établissement ou du promoteur à prendre en charge les coûts en ressources humaines et financières (ainsi que l'infrastructure déjà en place, comme l'existence d'un système de billetterie).

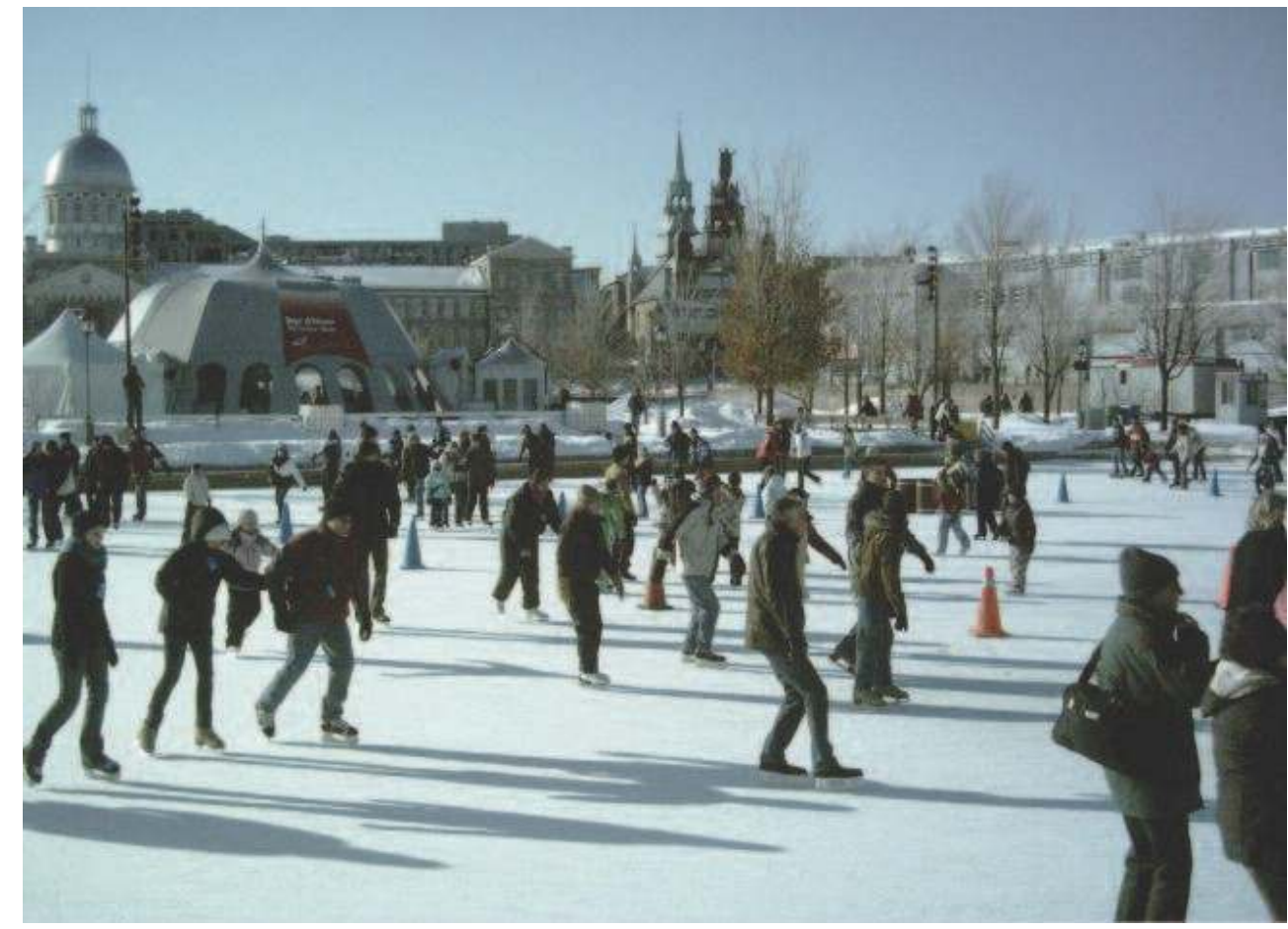

Patinoire au Vieux-Port de Montréal en février 2009

(C) Jean Gagnon

\section{La méthodologie de l'estimation de la fréquentation des Quais du Vieux-Port de Montréal}

Le territoire des Quais du Vieux-Port est un site extérieur de grande étendue dont le niveau de fréquentation est tributaire des conditions météorologiques. Il dispose de six voies d'accès libre (sans contrôle d'accès ou barrières à l'entrée) et demeure ouvert au grand public tous les jours de l'année. On y offre une très grande diversité d'activités et d'animations qui s'adressent à une clientèle hétéroclite. La programmation, de nature souvent ponctuelle, influence parfois considérablement l'achalandage journalier mais néanmoins, elle dépend principalement de la saisonnalité. Il convient également de noter que le Centre des Sciences ainsi que les parcs de stationnement disposent d'un système de billetterie ou d'un système d'accès automatisé; cependant, les données issues de ces systèmes ne peuvent pas rendre compte de l'ensemble de l'achalandage sur le site.

On peut facilement déduire que pour répondre aux besoins d'estimation de l'achalandage des Quais du Vieux-Port, il fallait adopter une approche méthodologique qui puisse tenir compte de toutes les spécificités précédemment décrites et qui serait une combinaison des différentes méthodes d'évaluation existantes (voir le tableau page suivante). 


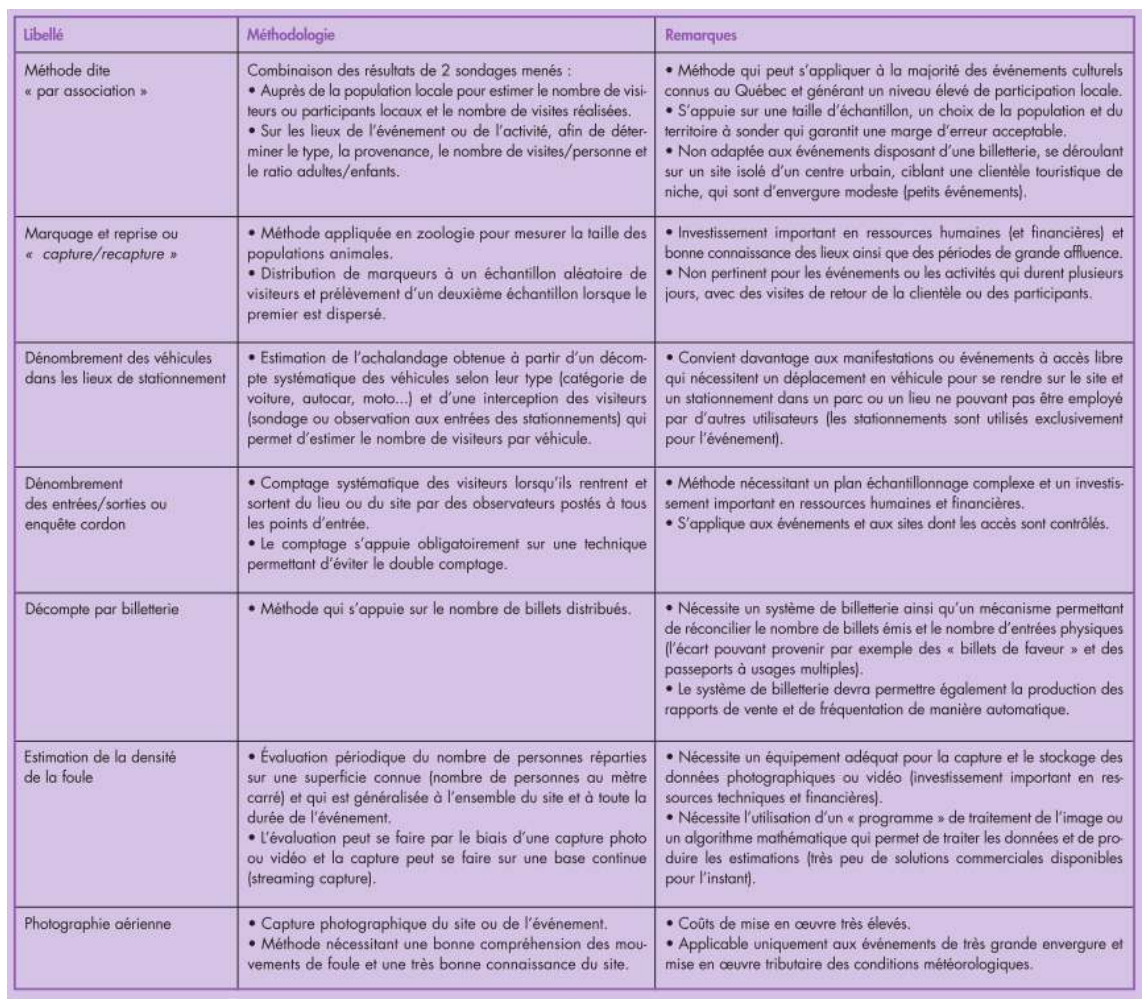

Aperçu des principales méthodes d'évaluation de l'achalandage

Source : Ministère du Tourisme, Québec, 2009

Compte tenu des nombreuses particularités et contraintes du site, la SVPM a décidé de confier le mandat de trouver la meilleure méthodologie d'estimation de l'achalandage (sur une base journalière) à une firme externe qui possède une expertise dans la modélisation de l'achalandage et qui jouit d'une bonne expérience dans le secteur touristique. Le recours à une firme privée indépendante garantissait l'aspect impartial et objectif des données produites et mettait le SVPM à l'abri d'éventuelles contestations sur la fiabilité des estimés et de la méthodologie.

Dans le cadre d'une démarche conjointe, la firme et la SVPM ont défini les intrants (ou variables) nécessaires au modèle d'estimation en essayant de tenir compte de l'ensemble des paramètres qui peuvent influencer

l'achalandage sur le site. Parallèlement à ce travail de réflexion sur les variables déterminantes, la firme a mené une série d'observations, de sondages auprès des visiteurs et de comptages systématiques sur le site. De plus, un inventaire des données disponibles à l'interne a été dressé (rapports de vente et de fréquentation extraits de la billetterie et des lieux de stationnement) en mettant l'accent sur la qualité des données (fiabilité, accessibilité, disponibilité, pérennité de la source). Par ailleurs, un processus cadre spécifique (voir le schéma page suivante) a été mis en place afin de clarifier les rôles des intervenants et de comprendre leur interrelation au sein de la démarche méthodologique. 


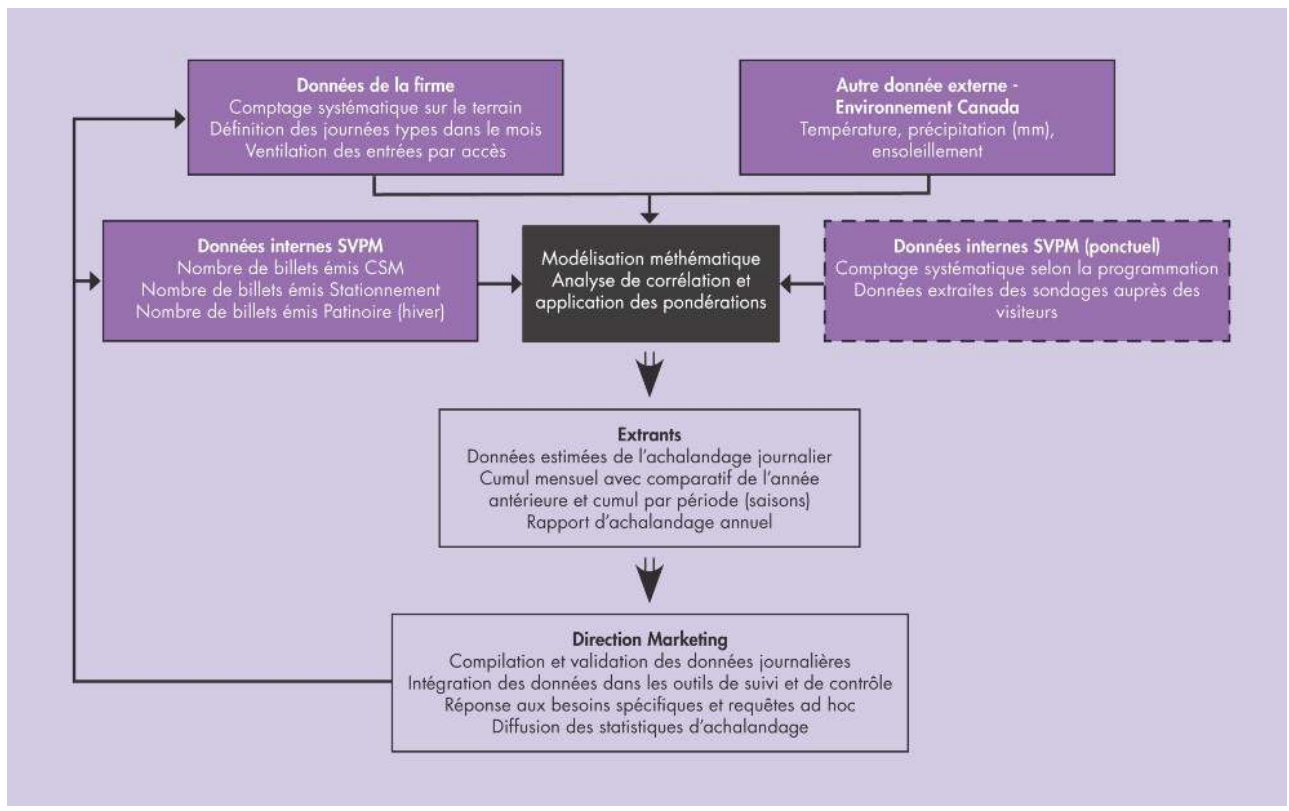

Aperçu des principales méthodes d'évaluation de l'achalandage

Source : Ministère du Tourisme, Québec, 2009

\section{Mise en œuvre et pérennisation du modèle}

\section{Un modèle dynamique qui évolue avec le temps}

Depuis sa mise en œuvre à la fin des années 1990, le modèle a subi des mises à jour afin qu'il puisse conserver sa fiabilité et sa pertinence. Si le cadre conceptuel du modèle est demeuré inchangé (garantissant de ce fait une comparabilité des données historiques), il a été nécessaire d'ajuster certains paramètres au niveau des intrants, notamment pour tenir compte de plusieurs facteurs, dont les suivants :

- les changements de comportement sur le plan de la fréquentation du site par les visiteurs (par exemple, constat d'une augmentation de l'achalandage en hiver au cours des cinq dernières années) ;

- la bonification de la programmation (incluant l'ajout de nouveaux intervenants commerciaux) qui s'oriente vers un site récréotouristique quatre saisons ;

- une programmation en soirée qui prend de l'expansion sur les Quais (ce qui implique la nécessité d'ajuster les périodes d'observation et de comptage sur le site);

- l'effet du changement climatique (hiver plus doux, plus de précipitations en été) ;

- une redynamisation du secteur résidentiel du quartier et de nouveaux projets d'aménagement urbain (augmentation des résidents de proximité et impact sur la ventilation du volume d'entrées aux points d'accès);

- une plus grande conscience environnementale au sein de la population (impact sur le choix du transport).

Par ailleurs, le modèle ayant été conçu pour être flexible, l'ajout ou la bonification des données des comptages systématiques ponctuels lors des événements de programmation d'envergure, notamment la Fête du Canada, les spectacles nautiques, les concerts musicaux et les autres activités culturelles, permet d'obtenir des estimés en adéquation avec la réalité de la programmation et ainsi d'augmenter la fiabilité des résultats obtenus. 


\section{L'engagement organisationnel} l'achalandage, l'organisation doit continuer à soutenir de manière continue les coûts de fonctionnement liés à l'exploitation et la mise à jour du modèle. Pour la SVPM, cela s'est notamment traduit par un engagement à long terme avec la firme (près de 10 ans actuellement) et par un budget annuel récurrent alloué à l'estimation de l'achalandage.

La SVPM retire trois principaux avantages en établissant cette relation à long terme avec la firme et en assurant la pérennité du modèle :

- le renforcement progressif de la fiabilité du modèle au fil du temps en raison de la stabilité de la méthodologie utilisée ;

- la constitution d'une série chronologique statistiquement fiable pour déceler des tendances (en termes de fluctuation) et permettre des analyses comparatives selon des périodes ou des dates précises;

- mais surtout, avec près de 10 ans de données dans la base de données, l'établissement des prévisions sur l'achalandage à court et moyen terme, un atout indéniable qui peut grandement influer sur la planification et la mise en marché de l'offre de programmation sur le site.

\section{L'utilisation des données d'achalandage au quotidien}

L'exploitation de manière pragmatique des estimés mensuels d'achalandage peut être illustrée par une brève description du traitement des données par le service de marketing.

Dès leur réception, les données sont intégrées au tableau de bord de marketing. Le résultat mensuel est comparé à celui de l'année précédente (même mois) mais également à l'objectif mensuel (initialement établi par le bais d'un modèle prévisionnel sur les données historiques). Le cumul depuis le début de l'année jusqu'au dernier mois écoulé (avec le comparatif de l'année précédente, pour la même période) est également présenté. Une analyse est effectuée par la suite pour expliquer les écarts observés (positifs ou négatifs).

21 Les données d'achalandage sont croisées avec les données du sondage auprès des visiteurs pour permettre de déterminer la ventilation de la provenance et de la langue des visiteurs du mois observé. Les pourcentages obtenus sont comparés avec les objectifs fixés en termes de taux de pénétration du marché géographique et de taux de capture de la clientèle anglophone et francophone. Les données sont également transmises (sur une base mensuelle ou selon une période ou une date particulière dans le mois) aux responsables de la programmation afin d'alimenter le bilan de chaque activité offerte durant le mois observé.

Après validation et analyse, les données mensuelles sont communiquées à l'office du tourisme régional (Tourisme Montréal) ${ }^{6}$ et au regroupement des attraits touristiques au Québec (SATQ) ${ }^{7}$ en vue d'alimenter la base de données des attraits touristiques et de permettre une comparaison avec l'industrie (portrait de la concurrence et tendance de l'industrie). 


\section{NOTES}

1. Le Centre des Sciences de Montréal appartient à la catégorie des centres d'interprétation à caractère scientifique selon la nomenclature de l'observatoire de la Culture du Québec, organisme rattaché à l'Institut de la Statistique du Québec.

2. Tourisme Montréal, 2009.

3. Sondage auprès des clientèles pour le Vieux-Montréal, Léger Marketing pour la Société de développement de Montréal, octobre 2004.

4. Le taux de notoriété peut être comparé à une cote de popularité d'institutions ou de lieux (sites récréotouristiques, par exemple) de même nature ou de vocation/mission similaire selon la population ou les visiteurs. La mesure se base sur la reconnaissance spontanée et assistée des répondants au moyen d'un sondage mené par une firme indépendante.

5. ibid note 4 .

6. Consulter notamment la base de données de Tourisme Montréal sur www.tourismemontreal.org/Tourisme-Montreal/Trousse-d-outils

7. Consulter le site du regroupement sur www.attractionsquebec.qc.ca/ accueil.aspx

\section{RÉSUMÉS}

Dans cette période de regroupement collectif, une question demeure pour tout analyste: comment compter une foule de personnes à un événement ouvert, qui n'impose pas un circuit prédéfini ? Quelles sont les méthodes applicables à nos manifestations culturelles comme la fête de la science ? L'exemple nous vient du côté du Québec, de la Société du Vieux Port de Montréal, qui présente ici une méthode - basée sur une modélisation mathématique et des observations in situ - pour mesurer l' "achalandage" selon leur expression à prendre dans le sens de fréquentation, $\mathrm{du}$ site $\mathrm{du}$ vieux port et comment ce suivi induit un certain type de programmation culturelle. 
INDEX

Mots-clés : étude de publics, évaluation, Fête de la Science, indicateurs, marketing, observation, programmation culturelle

Index géographique : Canada, Montréal

\section{AUTEUR}

HAJA RAMAHATRA

Analyste marketing au Centre des Sciences de Montréal 